\title{
Magnetic quantum oscillations in borocarbide superconductors
}

\author{
B. Bergk and J. Wosnitza \\ Hochfeld-Magnetlabor Dresden (HLD), Forschungszentrum Dresden-Rossendorf, Dresden D-01314, Germany \\ E-mail: j.wosnitza@ffzd.de
}

Received April 7, 2009

\begin{abstract}
We report systematic de Haas-van Alphen (dHvA) investigations in the normal and superconducting state of $\mathrm{RNi}_{2} \mathrm{~B}_{2} \mathrm{C}(\mathrm{R}=\mathrm{Y}$ and $\mathrm{Lu})$. The observed rich frequency spectrum of the dHvA signals results from a rather complex electronic band structure with different open and closed Fermi-surface sheets. From our data in combination with full-potential local-orbital calculations we are able to extract the angular-resolved mass-enhancement factors, $\lambda$, for different bands. We find a strong anisotropy and band dependence of $\lambda$, clearly reflecting the multiband character of the superconductivity in $\mathrm{RNi}_{2} \mathrm{~B}_{2} \mathrm{C}$. We further were able to resolve dHvA oscillations deep into the superconducting state. The observed additional damping of the dHvA amplitudes is much less than expected from most theories. This hints at a reduced or even zero superconducting gap for the detected Fermi surface.
\end{abstract}

PACS: 74.70.Dd Ternary, quaternary, and multinary compounds;

71.18.+y Fermi surface: calculations and measurements; effective mass, $g$ factor; 74.25.Jb Electronic structure.

Keywords: superconducting state, borocarbide, oscillations, Fermi surface.

\section{Introduction}

One of the most powerful methods to determine bulk electronic band-structure properties of metals and superconductors is the measurement of magnetic quantum oscillations. From that, the Fermi-surface topology, band-resolved effective masses, scattering times, and other band-structure parameters are obtainable. In combination with state-of-the-art band-structure calculations the electronic properties including many-body effects can be investigated in great detail.

The mostly used experimental technique is the detection of the de Haas-van Alphen (dHvA) effect, i.e., measuring the field-dependent oscillations in the magnetization. For that, a well developed and highly accurate semi-classical theoretical description, the so-called Lifshitz-Kosevich (LK) theory, is available $[1,2]$. By use of this technique important information on materials even with rather involved band structures, such as in $\mathrm{RNi}_{2} \mathrm{~B}_{2} \mathrm{C}$, can be gained. $\mathrm{R}$ stands for a rare-earth ion and here in especially for $\mathrm{Y}$ and $\mathrm{Lu}$. For that, highly sophisticated efforts both from the experimental as well as from the theoretical side are needed.
Even 15 year after the discovery of superconductivity in the quaternary borocarbides $[3,4]$ this material class remains fascinating because of the possible coexistence of magnetism and superconductivity for some rare-earth ions. But even for the nonmagnetic superconductors $\mathrm{YNi}_{2} \mathrm{~B}_{2} \mathrm{C}$ and $\mathrm{LuNi}_{2} \mathrm{~B}_{2} \mathrm{C}$ certain features of the superconducting state are still unclear and, therefore, a matter of debate. Although many results point to a simple $s$-wave phonon-mediated superconductivity (see e.g. [5,6]), recent thermodynamic [7], tunneling [8], ultrasound [9], and point-contact measurements [10] suggest other scenarios, such as anisotropic $s$ - or $d$-wave, or $(s+g)$-wave pairing. Furthermore, there is an increasing evidence for multiband superconductivity in these materials with different and even anisotropic gaps for the various bands [11-18]. The rather involved crystal structure of $\mathrm{YNi}_{2} \mathrm{~B}_{2} \mathrm{C}$ and $\mathrm{LuNi}_{2} \mathrm{~B}_{2} \mathrm{C}$ (Fig. 1) leads to a complicated band structure [17,19-22] which supports this notion. In order to gain more insight in which bands are mainly involved in the superconductivity, dHvA experiments in combination with modern band-structure calculations are a preferable choice. Here, we summarize our results recently obtained for $\mathrm{YNi}_{2} \mathrm{~B}_{2} \mathrm{C}$ and $\mathrm{LuNi}_{2} \mathrm{~B}_{2} \mathrm{C}$. 


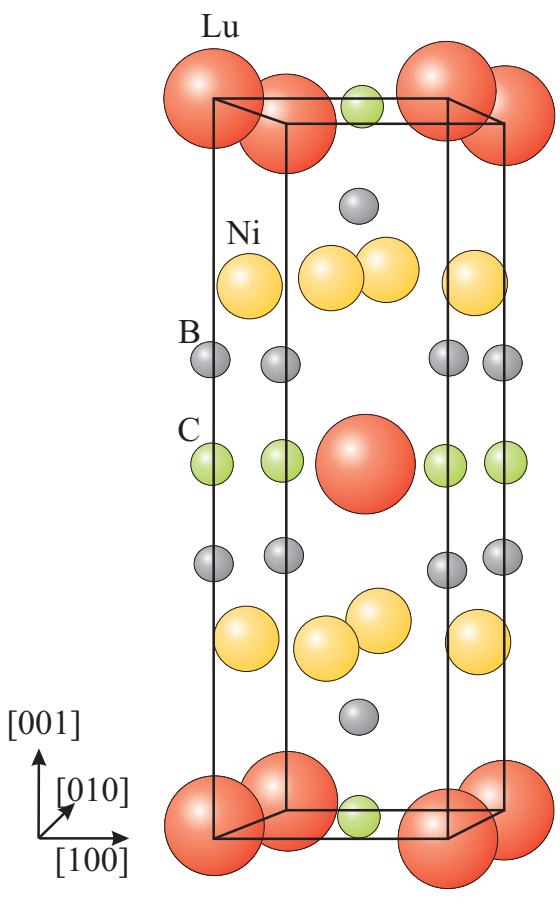

Fig. 1. Crystal structure of $\mathrm{LuNi}_{2} \mathrm{~B}_{2} \mathrm{C}$.

\section{Experimental}

We investigated $\mathrm{YNi}_{2} \mathrm{~B}_{2} \mathrm{C}$ single crystals prepared by two different methods. Part of the crystals was grown by a zone-melting technique as described in Ref. 23, improved by optical heating. This resulted in large crystals of a few $\mathrm{cm}^{3}$ size from which smaller parts were cut for our investigations. The high crystal quality was evident from a high residual resistivity ratio [24] as well as from $\mathrm{x}$-ray diffraction and metallographic methods, such as electron-beam microanalysis. The other part of the $\mathrm{YNi}_{2} \mathrm{~B}_{2} \mathrm{C}$ crystals was prepared by a flux-growth technique as described in [25]. Here, we show only data for a crystal grown by the latter method. Both types of crystals show the same superconducting transition temperature at $T_{c}=$ $=15.1(1) \mathrm{K}$. The dHvA signals of both crystal types in the normal state agrees nicely with each other verifying similar crystal qualities as reflected by comparable scattering rates. Only the damping of the dHvA oscillations in the superconducting state differs largely. Whereas the zone-melted crystals revealed an abrupt vanishing of the dHvA signal below the upper critical field, $B_{c 2}$ [24], the dHvA oscillations in the flux-grown crystals persisted down to rather low fields, very much as observed for the $\mathrm{LuNi}_{2} \mathrm{~B}_{2} \mathrm{C}$ crystals discussed below. Although we can only speculate on the origin of this different damping behavior, it might be related to different flux-pinning mechanisms and, therefore, different internal magnetic-field inhomogeneities (see the discussion below). The
$\mathrm{LuNi}_{2} \mathrm{~B}_{2} \mathrm{C}$ single crystals were as well flux grown resulting in platelets of a few $\mathrm{mm}^{3}$ size (onset $T_{c}=16.5 \mathrm{~K}$ ) [25].

The dHvA oscillations were measured either by use of a capacitive cantilever torquemeter or using the modulation-field technique. The latter method was mainly utilized to study the dHvA signals in the superconducting state. These measurements were performed at the HLD in Dresden using a ${ }^{3} \mathrm{He}$ cryostat equipped with a $15 \mathrm{~T}$ superconducting magnet. The torque measurements were also done at the HLD up to $15 \mathrm{~T}$ and up to about $32 \mathrm{~T}$ at the Grenoble High Magnetic Field Laboratory (GHMFL) and at the High Field Magnetic Laboratory in Nijmegen. A dilution refrigerator as well as ${ }^{3} \mathrm{He}$ cryostats have been used to cover the temperature range from $20 \mathrm{mK}$ up to about $10 \mathrm{~K}$. All samples were placed on cantilever platforms which could be rotated in situ.

\section{Results and Discussion}

A typical torque signal measured at $T=50 \mathrm{mK}$ at the GHMFL is shown in Fig. 2, $a$. In the superconducting state, below $B_{c 2} \approx 8.3 \mathrm{~T}$ a large hysteresis between up and down sweep is apparent. Just before the normal state is reached, a pronounced peak appears in the up-sweep torque signal. This feature, known as the peak effect [26], has been observed with different magnitudes for all angles and for all investigated $\mathrm{RNi}_{2} \mathrm{~B}_{2} \mathrm{C}$ crystals $[24,27,28]$. Although the details of this phenomenon are not fully understood, it has been observed for a number of type-II superconductors and is attributed to distinct vortex-matter phases with intrinsically different pinning strengths [26].
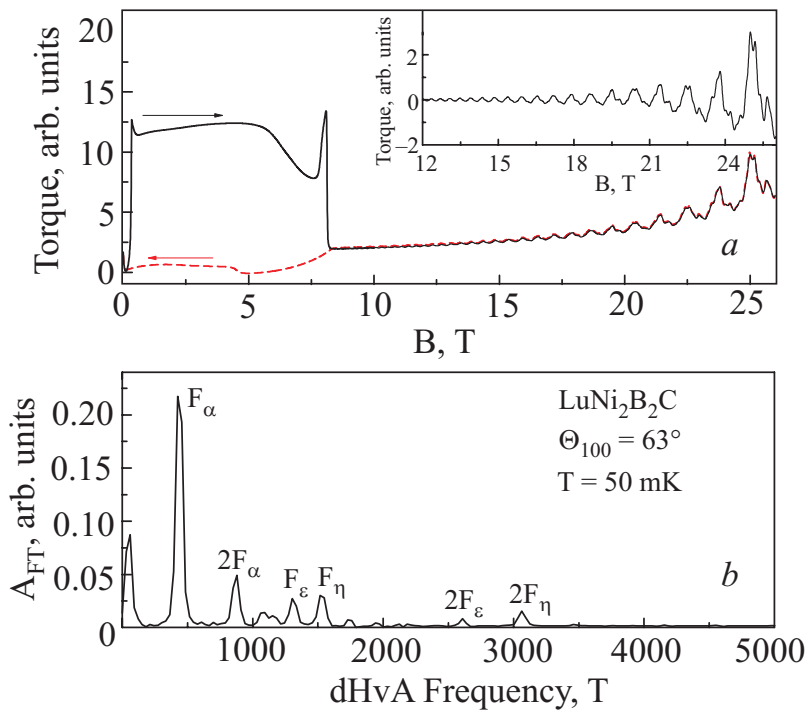

Fig. 2. Field dependence of the torque signal of $\mathrm{LuNi}_{2} \mathrm{~B}_{2} \mathrm{C}$ with the magnetic field rotated $63^{\circ}$ from the $c$ axis towards [100]. The arrows indicate the field-sweep directions. The inset shows the torque signal after background subtraction $(a)$. Fourier spectrum of the signal shown in the inset $(b)$. 
Above the upper critical field, $B_{c 2}$, clear dHvA oscillations become visible, which can be better resolved in the inset of Fig. 2, $a$, where the background torque signal has been subtracted. The dominant oscillation frequency, $F_{\alpha} \approx 440 \mathrm{~T}$ (see the Fourier transformation in Fig. 2,b), originates from a spheroidal Fermi surface. The quasiparticles of the band forming this Fermi surface have a reduced effective mass, $m_{\mathrm{eff}}^{\alpha}$, between 0.3 and 0.45 $m_{e}$ depending on the field orientation, where $m_{e}$ is the free-electron mass [17]. dHvA oscillations originating from this Fermi surface were clearly observed for all crystallographic orientations. The found angular dependence of $F_{\alpha}$ reflects the spheroidal shape and agrees with bandstructure calculations (Figs. 3 and 4). For details on the sophisticated fully relativistic full-potential local-orbital band-structure calculations see Ref.17. The calculated $F_{\alpha}$ is, however, shifted upwards by about $400 \mathrm{~T}$. Although that appears to be a large deviation, this can be explained by the very small contribution (less than 1 procent) of the corresponding band to the total density of states at the Fermi energy. By shifting the band filling by a tiny amount as low as $0.012 e^{-}$, excellent agreement with the dHvA data can be reached. The change of the dHvA frequencies of the other Fermi surfaces would be negligible. The small density of states together with a small mass renormalization on the spheroidal Fermi surface (see below) [17] reflects the minor relevance of this band for superconductivity in $\mathrm{LuNi}_{2} \mathrm{~B}_{2} \mathrm{C}$.

Besides the frequency $F_{\alpha}$, the Fourier-transformed dHvA signal shows a rich frequency spectrum (Fig. 2). For the shown angle, two other independent oscillation frequencies appear, $F_{\varepsilon}$ and $F_{\eta}$, which can be ascribed to a cube-like and cushion-like Fermi surface originating from one other band (Fig. 3) [17]. The other peaks in the Fourier transformation are the second harmonics of the mentioned $\mathrm{dHvA}$ frequencies. The effective masses of the $\varepsilon$ and $\eta$ orbit are larger (between about 1 and 2 free-electron masses) than for the $\alpha$ orbit. Consequently, much higher magnetic fields are needed to resolve these oscillations.

The dHvA-frequency spectra at other field orientations become even richer (Fig. 4). As a further example, the background- subtracted torque signal for the magnetic field aligned along the [100] direction together with the Fourier-transformed spectrum is shown in Fig. 5, $a$ for $\mathrm{LuNi}_{2} \mathrm{~B}_{2} \mathrm{C}$. For this angle some smaller peaks in the Fourier transformation appear next to the frequency $F_{\alpha}$. Their origin is unclear, but they most likely result from more evolved, e.g., corrugated, Fermi-surface topologies for the different bands.

Besides the already introduced dHvA frequencies, $F_{\alpha}$, $F_{\varepsilon}$, and $F_{\eta}$, a new orbit gives rise to the strong peak at about $5260 \mathrm{~T}$, labeled $F_{x}$. This orbit could not be assigned directly to an extremal orbit of the calculated Fermi surface, but is very likely caused by the compli-
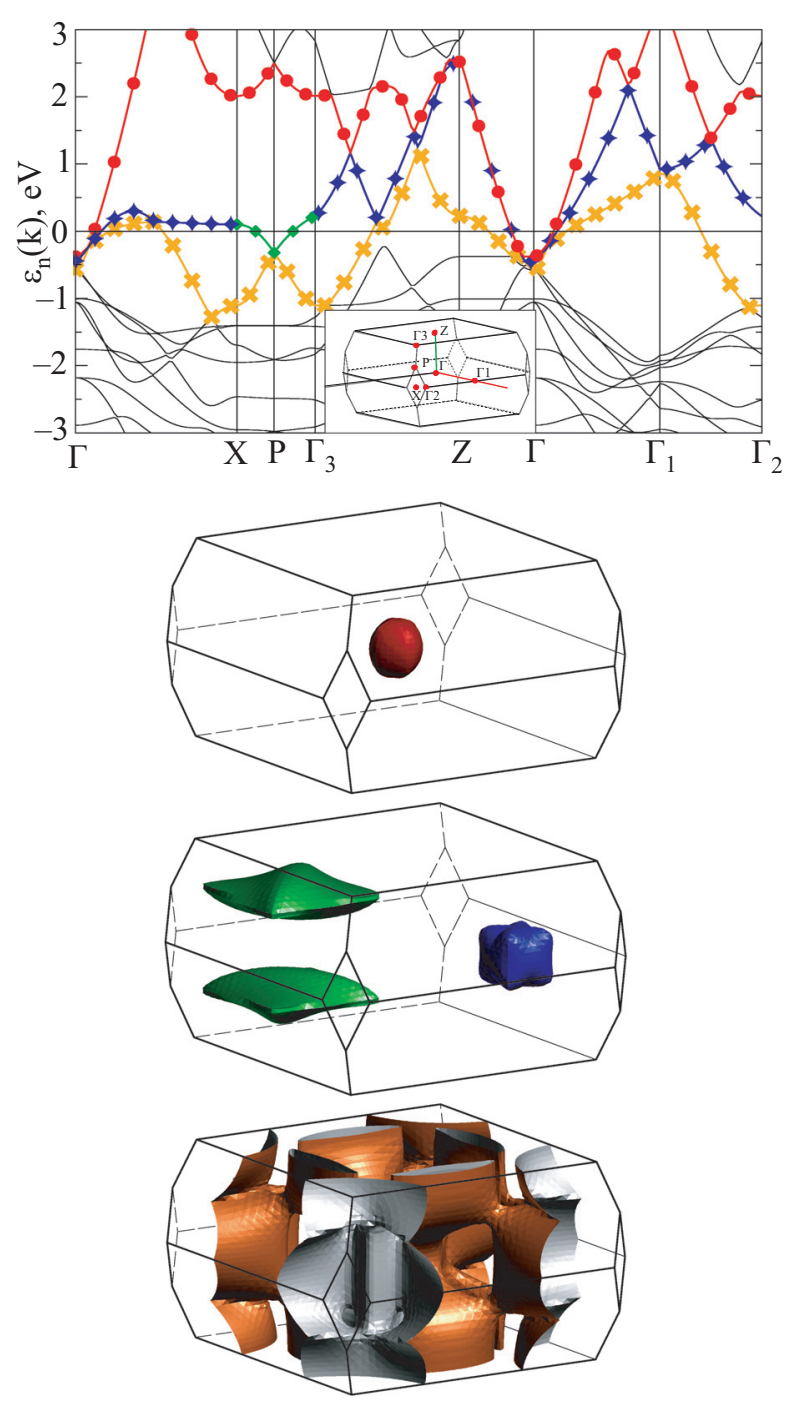

Fig. 3. Calculated band structure (upper panel) and corresponding Fermi surfaces of $\mathrm{LuNi}_{2} \mathrm{~B}_{2} \mathrm{C}$. The bands are labeled according to the resulting Fermi surfaces: the band highlighted by circles $(\bullet)$ results in the spheroidal, diamonds $(\star)$ give the cube-like, rhombs $(\diamond)$ give rise to the cushion-like, and crosses (X) result in the branched Fermi-surface. The cube-like and cushion-like Fermi surfaces in the third panel are shifted by $(\pi / a$, $\pi / a, 0)$ to present simply connected sheets. The high-symmetry points are given in the inset of the upper panel. Reproduced from Ref. 17.

cated branched Fermi surface (Fig. 3) [17]. Isshiki et al. have observed this frequency, too [29]. They called the frequency $F_{\Phi}$ and assigned it to an extremal orbit of the mentioned branched Fermi surface.

For direct comparison, the dHvA signal of $\mathrm{YNi}_{2} \mathrm{~B}_{2} \mathrm{C}$ measured at the same temperature and for the same field orientation is shown in Fig. 5,b, together with its Fourier transformation. A very similar dHvA signal and spectrum are observed; the peaks in the Fourier spectrum at $F_{\alpha}, F_{\eta}$, and $F_{x}$ can be ascribed to equivalent orbits as for 


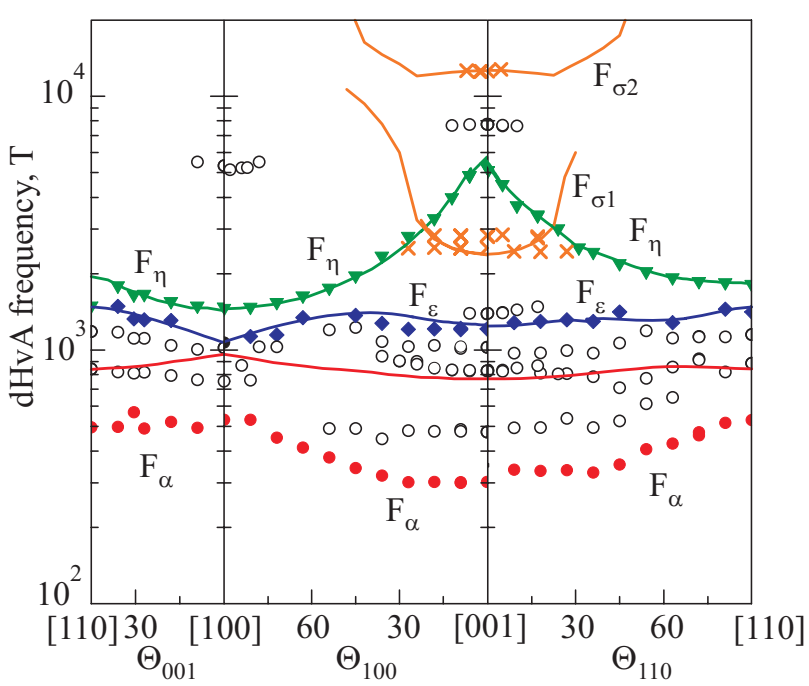

Fig. 4. Angular dependence of the dHvA frequencies in $\mathrm{LuNi}_{2} \mathrm{~B}_{2} \mathrm{C}$. The solid lines are obtained by use of band-structure calculations [17]. The calculated dHvA frequency for the spherical Fermi surface, $F_{\alpha}$, is somewhat larger than measured, whereas for the other Fermi surfaces excellent agreement between theory and experiment is found. For the open circles no corresponding Fermi surface could be assigned unambiguously.

$\mathrm{LuNi}_{2} \mathrm{~B}_{2} \mathrm{C}$. The first double peak at about $900 \mathrm{~T}$ in the Fourier spectrum, labeled $F_{\alpha} / F_{\varepsilon}$, presumably comprises besides the $\alpha$ orbit also the $\varepsilon$ orbit. This assignment is, however, not unambiguously possible, since the $\alpha$ orbit in $\mathrm{YNi}_{2} \mathrm{~B}_{2} \mathrm{C}$ is somewhat larger and the $\varepsilon$ orbit seems to be somewhat smaller compared to the orbits in $\mathrm{LuNi}_{2} \mathrm{~B}_{2} \mathrm{C}$. Altogether, the extremal areas of the corresponding Fermi surfaces differ only slightly for the two materials. For $\mathrm{YNi}_{2} \mathrm{~B}_{2} \mathrm{C}$, the frequency $F_{x}$ has also been labeled $F_{\lambda}$ in Ref. 24 and presumably originates in the $\Phi$ orbit as shown in the calculated band structure for $\mathrm{YNi}_{2} \mathrm{~B}_{2} \mathrm{C}(\mathrm{Fig}$. 2 in Ref. 30). Our experimental results for $\mathrm{YNi}_{2} \mathrm{~B}_{2} \mathrm{C}$ fit well with data reported previously [31-36].

The apparent similarity of the Fermi-surface topologies of the two nonmagnetic borocarbides $\mathrm{YNi}_{2} \mathrm{~B}_{2} \mathrm{C}$ and $\mathrm{LuNi}_{2} \mathrm{~B}_{2} \mathrm{C}$ is reflected among other properties in their comparable $T_{c}$ values of 15.1 and $16.5 \mathrm{~K}$, respectively. This hints at very similar interaction strengths for the Cooper pairing in these superconductors. These coupling strengths should be reflected in the mass enhancements of the different bands. For $\mathrm{LuNi}_{2} \mathrm{~B}_{2} \mathrm{C}$, we have investigated this mass enhancement in great detail by use of temperature-dependent dHvA studies in combination with band-structure calculations applying a full-potential local-orbital code [37] in its scalar-relativistic version within the local density approximation $[17,18]$.

From the temperature dependences of the dHvA oscillation amplitudes, $A_{F T}$, the effective masses, $m_{\mathrm{eff}}$, including all many-body renormalizations can be extracted for
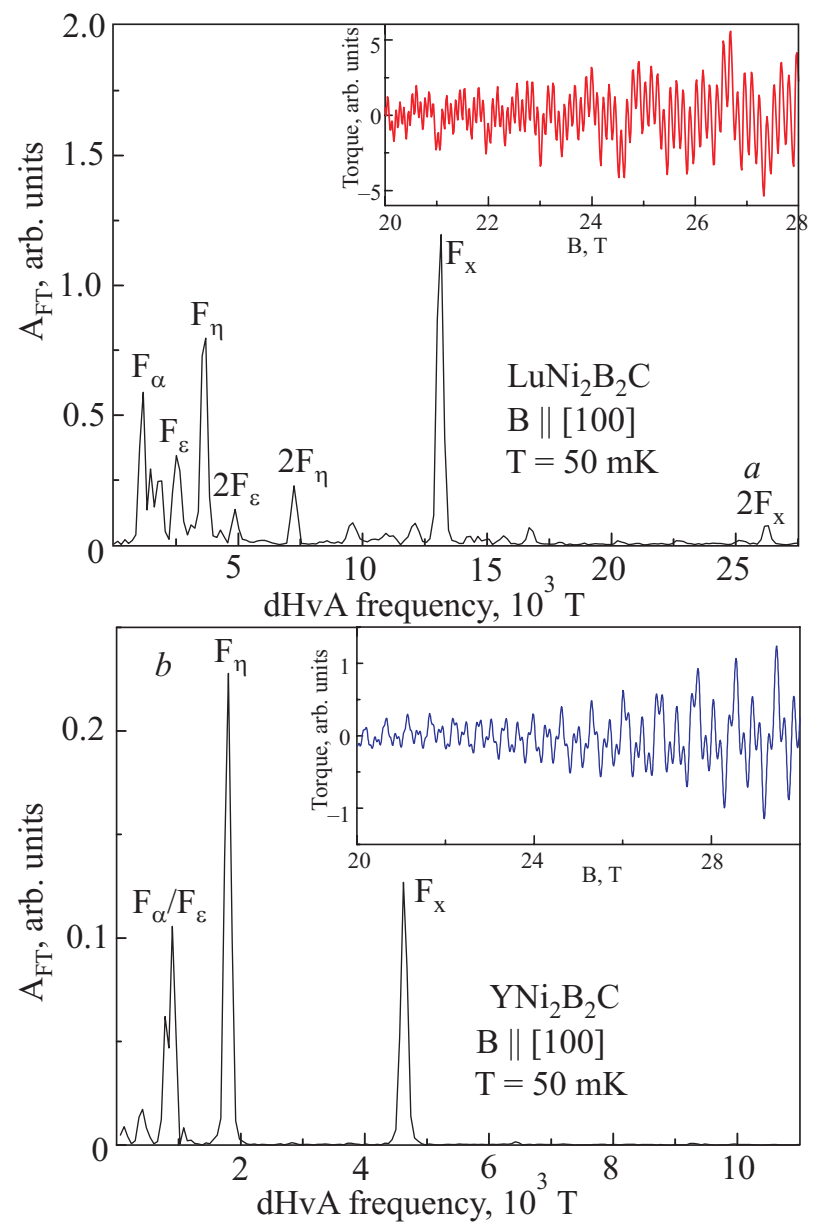

Fig. 5. The inset shows the field dependence of the torque signal of $\mathrm{LuNi}_{2} \mathrm{~B}_{2} \mathrm{C}$ after background subtraction with the magnetic field aligned along [100]. The main panel shows the Fourier spectrum of the signal $(a)$. The same for $\mathrm{YNi}_{2} \mathrm{~B}_{2} \mathrm{C}(b)$.

each orbit separately $[1,2]$. This is shown in Fig. 6 for the orbits $F_{\sigma 1}$ and $F_{\sigma 2}$. These orbits belong to the branched Fermi surface (Fig. 3). $F_{\sigma 1}$ could be ascribed to a windmill-shaped orbit and $F_{\sigma 2}$ to a lemon-like orbit [17]. The temperature-dependent dHvA-amplitude reduction is described by the LK damping factor $R_{T}=X / \sinh (X)$, with $X=\alpha m_{\text {eff }} T / B$ and $\alpha=2 \pi^{2} k_{B} m_{e} / e \hbar=14.69 \mathrm{~T} / \mathrm{K}$. The solid lines in Fig. 6 are fits using this function. From this two-parameter fit for each curve, we obtain similar effective masses of 3.5(2) and 3.6(3) $m_{e}$ for both orbits of the branched Fermi surface. Since in the calculated effective masses, $m_{\text {calc }}$, no electron-phonon effects are included, the angular-resolved mass enhancement factor, $\lambda=m_{\text {eff }} / m_{\text {calc }}-1$, can be extracted directly for each ascribed orbit. For the main crystallographic directions, the results are summarized in Table 1.

For the nonmagnetic borocarbides renormalizations of nonphononic origin are expected to be weak. This is evidenced by point-contact spectroscopy measurements $[38,39]$ as discussed in more detail in Ref. 17. Conse- 


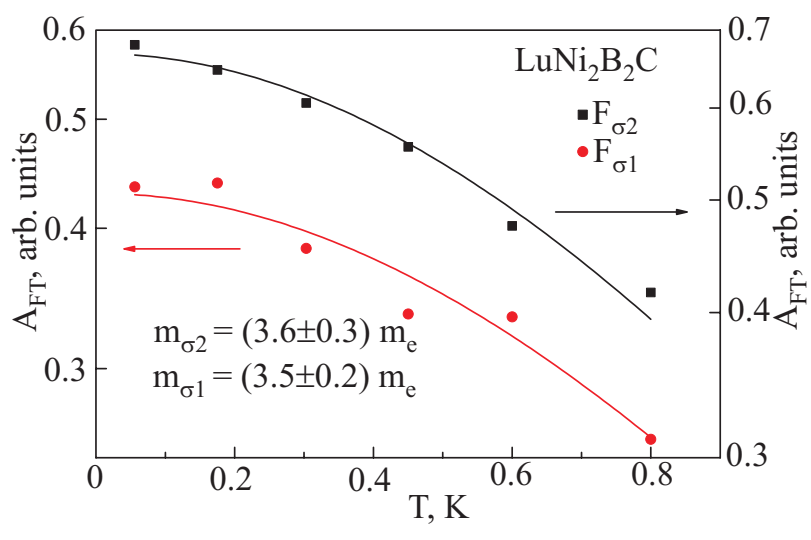

Fig. 6. Temperature dependence of the oscillation amplitudes of the lemon and windmill extremal areas of the branched Fermi surface with the respective fits by use of the Lifshitz-Kosevich theory.

Table 1. Band structure parameters, such as dHvA frequency $F$, effective mass $m_{\mathrm{eff}}$, in units of the free-electron mass $m_{e}$, calculated effective mass without renormalization $m_{\text {calc }}$, and the mass-enhancement factor, $\lambda$, for the high-symmetry directions of $\mathrm{LuNi}_{2} \mathrm{~B}_{2} \mathrm{C}$

\begin{tabular}{c|c|c|c|c|c}
\hline \hline Direction & Orbits & $F(T)$ & $m_{\mathrm{eff}}\left(m_{e}\right)$ & $m_{\text {calc }}\left(m_{e}\right)$ & $\lambda$ \\
\hline \hline$[100]$ & $\alpha$ & 530 & 0.47 & 0.3 & 0.6 \\
& $\varepsilon$ & 1075 & 0.8 & 0.4 & 1 \\
& $\eta$ & 1480 & 1.68 & 0.9 & 0.9 \\
{$[110]$} & $\alpha$ & 520 & 0.44 & 0.26 & 0.7 \\
& $\varepsilon$ & 1440 & 2 & 0.62 & 2.2 \\
& $\eta$ & 1820 & 1.9 & 1.55 & 0.23 \\
& $\alpha$ & 300 & 0.29 & 0.22 & 0.32 \\
& $\varepsilon$ & 1230 & 2.3 & 0.62 & 2.7 \\
& $\eta$ & 5440 & 4.25 & 2.0 & 1.1 \\
& $\sigma 1$ & 2440 & 3.5 & 2.0 & 0.8 \\
& $\sigma 2$ & 12500 & 3.6 & 1.9 & 0.9 \\
\hline \hline
\end{tabular}

quently, the extracted $\lambda$ values are most likely directly related to the Cooper-pair coupling parameter. For all observed bands, we find prominent anisotropies and largely different coupling strengths. The weakest coupling is found for the spherical Fermi surface. This and the mentioned low contribution of this band to the total density of states proves the minor relevance of this band for superconductivity. Interestingly, the dHvA signal of the spherical Fermi surface is resolvable well below $B_{c_{2}}$ deep into the mixed state (see below). For the $\eta \mathrm{dHvA}$ frequency, originating from the cushion-like Fermi surface, $\lambda$ is about 1 in the (100) plane and decreases continuously to about 0.23 towards [110]. This Fermi surface is believed to allow superconductivity together with commensurate antiferromagnetism in the magnetic members of the borocarbide family. The $5 d$ states of the rare-earth atoms, that mediate the magnetic interaction between the localized $4 f$ magnetic moments, are not affecting this Fermi-surface, so that superconductivity may survive in the magnetic borocarbides [40].

For the $\mathrm{dHvA}$ frequency $\varepsilon$, resulting from the cube-like Fermi surface, the coupling strength is highly anisotropic reaching $\lambda \approx 2.7$ for $B$ aligned along the [100] direction down to $\lambda \approx 1$ for other directions. For both identified parts of the branched Fermi surface, we determine intermediate-strength coupling constants of 0.8 and 0.9 . Our data compare well with other results stating Fermi-surface-averaged coupling constants between 0.5 and 1.2 $[8,15,41]$. Our dHvA results give strong support for multiband superconductivity in the borocarbides as has been suggested in earlier work [11-16].

A direct way to study the field-dependent evolution of the superconducting energy gap is the measurement of dHvA oscillations below $B_{c 2}$. By opening of a gap, an additional damping of the dHvA oscillations is expected [42]. This has been evidenced for a number of type II superconductors $[42,43]$. For the nonmagnetic borocarbides $\mathrm{YNi}_{2} \mathrm{~B}_{2} \mathrm{C}$ and $\mathrm{LuNi}_{2} \mathrm{~B}_{2} \mathrm{C}$, however, highly controversial results have been reported [24,28,29,31-36]. This has been discussed as a consequence of the highly anisotropic and band-dependent coupling strengths (see above) leading to anisotropic multiple gaps. Another explanation, however, would be different internal field inhomogeneities due to flux-pinning effects. This would explain the mentioned different additional damping for crystals prepared via different routes as well as our recent findings for $\mathrm{LuNi}_{2} \mathrm{~B}_{2} \mathrm{C}$ discussed below.

In earlier work, we have measured the dHvA oscillations by use of the torque method [17,18,24,27,28]. This technique is advantageous at higher magnetic field, but looses sensitivity towards low fields, that are of interest here. We, therefore, measured the dHvA signal of $\mathrm{LuNi}_{2} \mathrm{~B}_{2} \mathrm{C}$ by use of the modulation-field technique which gains in sensitivity at lower magnetic fields [2]. By carefully using this method, we were able to observe the dHvA signal of the spherical Fermi surface for all crystallographic directions deep into the superconducting state. For some magnetic field orientations close to the [100] direction, we additionally could resolve the $\eta \mathrm{dHvA}$ frequency of the cushion-like Fermi surface in the mixed state.

As an example, Fig. 7 shows the measured modulation-field signal for up and down sweeps of the magnetic field. The dHvA oscillations become clearly visible after careful stepwise background subtractions (two upper panels of Fig. 7). The grey lines (shone in the upper panels) 


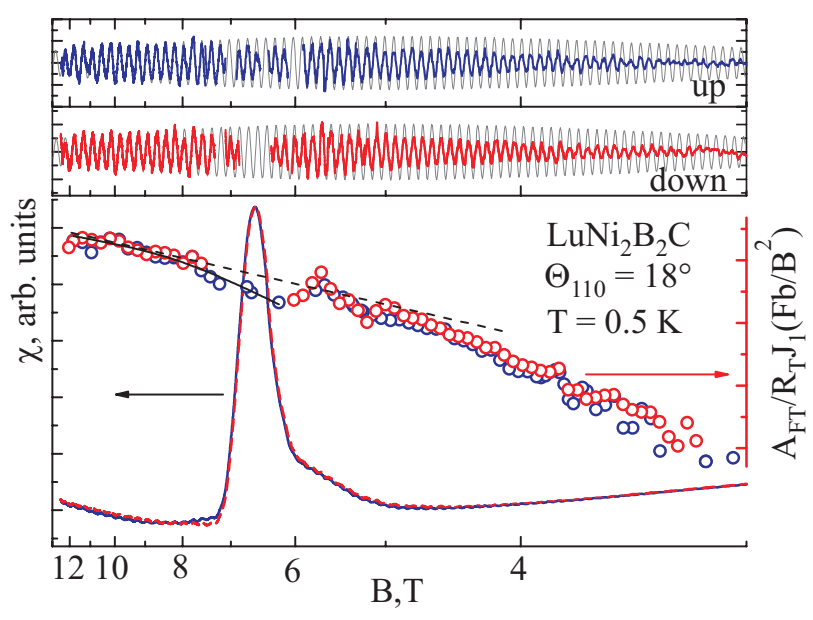

Fig. 7. Field dependence of the modulation-field signal of $\mathrm{LuNi}_{2} \mathrm{~B}_{2} \mathrm{C}$ shown in a $1 / B$ scale with the magnetic field rotated $18^{\circ}$ from the $c$ axis towards [100]. In the lower panel the as-measured signals for the up and down sweep are plotted (solid lines, left axis). In the two upper panels the dHvA signals after background subtraction are shown together with the calculated dHvA oscillations without additional damping in the superconducting state (thin grey lines). In the lower panel also the dHvA amplitudes extracted from the measured signals are presented in a Dingle plot (right axis). For such a plot from modulation-field signals the additional correction factor $J_{1}\left(F b / B^{2}\right)$ is needed. $J_{1}$ is the Bessel function of first order and $b$ is the modulation-field amplitude. The lines are explained in the text.

depict the dHvA signal without opening of a superconducting energy gap extrapolated from the normal state. In the Dingle plot (lower panel of Fig. 7, right axis) this extrapolated behavior is shown by the dashed line.

Just below $B_{c 2}$, we observe clearly an additional damping of the dHvA signal which is more or less restricted to the peak-effect region. We have described that field dependence of the dHvA amplitude by use of the well-accepted theory [44] taking into account fluctuations close to the superconducting transition [45]. With $B_{c 2}=8.2 \mathrm{~T}$, we obtain a zero-field energy gap of $\Delta=$ $=(3.0 \pm 0.5) \mathrm{meV}$. The relevance of this energy gap is, however, highly questionable since the dHvA signals clearly recovers towards lower magnetic fields below the peak effect (Fig. 7). Such kind of behavior cannot be described by use of the usual theories. These theoretical treatments are anyway mostly only applicable close to $B_{c 2}$. For $\mathrm{LuNi}_{2} \mathrm{~B}_{2} \mathrm{C}$, we can resolve $\mathrm{dHvA}$ signals in some cases down to $2.5 \mathrm{~T}$, way beyond any expectation for a gapped Fermi surface deep in the superconducting state.

It is, therefore, highly likely that the energy gap for the spherical Fermi surface in $\mathrm{LuNi}_{2} \mathrm{~B}_{2} \mathrm{C}$ is tiny or even not existing. The additional damping observed in the region of the prominent peak effect may be caused by large magnetic-field inhomogeneities. In this region a strongly changing flux-line lattice is expected which, therefore, should lead to an inhomogeneous internal magnetic field. Towards lower fields, a more equally distributed flux-line lattice is expected leading to a less inhomogeneous internal field. Towards very low fields the flux lines become more and more dilute and lead to an increasing field modulation of the internal magnetic field. Whether that is the reason for the observed small additional damping at low fields is so far unclear. Consequently, our results point to a very small or zero gap on one of the bands in $\mathrm{LuNi}_{2} \mathrm{~B}_{2} \mathrm{C}$.

\section{Summary}

We carefully measured the magnetic quantum oscillations of the nonmagnetic borocarbide superconductors $\mathrm{YNi}_{2} \mathrm{~B}_{2} \mathrm{C}$ and $\mathrm{LuNi}_{2} \mathrm{~B}_{2} \mathrm{C}$. In our comprehensive $\mathrm{dHvA}$ study we could resolve the rather involved band structure in combination with state-of-the-art band-structure calculations. By that, we obtained clear evidence for highly anisotropic band-dependent superconducting coupling strengths. For one band, our dHvA measurements in the superconducting state point to a tiny or non-existing energy gap.

\section{Acknowledgments}

We acknowledge P.C. Canfield and G. Behr for supplying the high-quality samples, H. Rosner, V. Petzold, and S.-L. Drechsler for their theoretical input, A. D. Bianchi, M. Bartkowiak, O. Ignatchik, I. Sheikin, and J. Perenboom for experimental support. We thank all of them for helpful discussions. The work was supported by the DFG through SFB 463. Part of this work has been supported by EuroMagNET under the EU contract RII3-CT-2004-506239 of FP6. The work at GHMFL was supported by the EC program Transnational Access - Specific Support Action (Contract No. RITA-CT-2003-505474). Work at the Ames Laboratory was supported by the Department of Energy, Basic Energy Sciences under Contract No. DE-AC02-07CH11358.

1. M. Lifshitz and A.M. Kosevich, Zh. Eksp. Teor. Fiz. 29, 730 (1955) [Sov. Phys. JETP 2, 636 (1956)].

2. D. Shoenberg, Magnetic Oscillations in Metals, Cambridge University Press, Cambridge (1984).

3. R.J. Cava, H. Takagi, H.W. Zandbergen, J.J. Krajewski, W.F. Peck, T. Siegrist, B. Batlogg, R.B. van Dover, R.J. Felder, K. Mizuhashi, O.J. Lee, H. Eisaki, and S. Uchida, Nature 367, 252 (1994).

4. T. Siegrist, H.W. Zandbergen, R.J. Cava, J.J. Krajewski, and W.F. Peck, Nature 367, 254 (1994).

5. T. Ekino, H. Fujii, M. Kosugi, Y. Zenitani, and J. Akimitsu, Physica C235-240, 2529 (1994).

6. R. Movshovich, M.F. Hundley, J.D. Thompson, P.C. Canfield, B.K. Cho, and A.V. Chubukov, Physica C227, 381 (1994). 
7. D. Lipp, M. Schneider, A. Gladun, S.-L. Drechsler, J. Freudenberger, G. Fuchs, K. Nenkov, K.-H. Müller, T. Cichorek, and P. Gegenwart, Europhys. Lett. 58, 435 (2002).

8. P. Martínez-Samper, H. Suderow, S. Viera, J.P. Brison, N. Luchier, P. Lejay, and P.C. Canfield, Phys. Rev. B67, 014526 (2003).

9. T. Watanabe, M. Nohara, T. Hanaguri, and H. Takagi, Phys. Rev. Lett. 92, 147002 (2004).

10. P. Raychaudhuri, D. Jaiswal-Nagar, G. Sheet, S. Ramakrishnan, and H. Takeya, Phys. Rev. Lett. 93, 146802 (2004).

11. S.V. Shulga, S.-L. Drechsler, G. Fuchs, and K.-H. Müller, Phys. Rev. Lett. 80, 1730 (1998).

12. N.L. Bobrov, S.L. Beloborod'ko, L.V. Tyutrina, I.K. Yanson, D.G. Naugle, and K.D.D. Rathnayaka, Phys. Rev. B71, 014512 (2005).

13. Yu.G. Naidyuk, D.L. Bashlakov, N.L. Bobrov, V.N. Chernobay, O.E. Kvitnitskaya, I.K. Yanson, G. Behr, S.-L. Drechsler, G. Fuchs, D. Souptel, D.G. Naugle, K.D.D. Rathnayaka, and J.H. Ross, Jr., Physica C460-462, 107 (2007).

14. S. Mukhopadhyay, G. Sheet, P. Raychaudhuri, and H. Takeya, Phys. Rev. B72, 014545 (2005).

15. S. Manalo, H. Michor, M. El-Hagary, G. Hilscher, and E. Schachinger, Phys. Rev. B63, 104508 (2001).

16. T. Yokoya, T. Baba, S. Tsuda, T. Kiss, A. Chainani, S. Shin, T. Watanabe, M. Nohara, T. Hanaguri, H. Takagi, Y. Takano, H. Kito, J. Itoh, H. Harima, and T. Oguchi, J. Phys. Chem. Solids 67, 277 (2006).

17. B. Bergk, V. Petzold, H. Rosner, S.-L. Drechsler, M. Bartkowiak, O. Ignatchik, A.D. Bianchi, I. Sheikin, P.C. Canfield, and J. Wosnitza, Phys. Rev. Lett. 100, 257004 (2008).

18. B. Bergk, V. Petzold, H. Rosner, S.-L. Drechsler, M. Bartkowiak, O. Ignatchik, I. Sheikin, P.C. Canfield, and J. Wosnitza, J. Phys.: Conf. Ser. 150, 052021 (2009).

19. L.F. Mattheiss, Phys. Rev. B49, 13279 (1994).

20. W.E. Pickett and D.J. Singh, Phys. Rev. Lett. 72, 3702 (1994).

21. H. Kim C.-D. Hwang and J. Ihm, Phys. Rev. B52, 4592 (1995).

22. S.B. Dugdale, M.A. Alam, I. Wilkinson, R.J. Hughes, I.R. Fisher, P.C. Canfield, T. Jarlborg, and G. Santi, Phys. Rev. Lett. 83, 4824 (1999).

23. G. Behr, W.Löser, G. Graw, H. Bitterlich, J. Freudenberger, J. Fink, and L. Schultz, J. Cryst. Growth 198/199, 642 (1999).
24. O. Ignatchik, T. Coffey, J. Hagel, M. Jäckel, E. Jobiliong, D. Souptel, G. Behr, and J. Wosnitza, J. Magn. Magn. Mater. 290-291, 424 (2005).

25. M. Xu, P.C. Canfield, J.E. Ostenson, D.K. Finnemore, B.K. Cho, Z.R. Wang, D.C. Johnston, Physica C227, 321 (1994).

26. M. Marchevsky, M.J. Higgins, and S. Bhattacharya, Nature 409, 591 (2001).

27. D. Bianchi, B. Bergk, O. Ignatchik, J. Wosnitza, J. Perenboom, and P.C. Canfield, J. Phys.: Conf. Ser. 51, 263 (2006).

28. B. Bergk, O. Ignatchik, A.D. Bianchi, M. Jäckel, J. Wosnitza, J. Perenboom, and P.C. Canfield, Physica C460-462, 630 (2007).

29. T. Isshiki, N. Kimura, H. Aoki, T. Terashima S. Uji, K. Yamauchi, H. Harima, D. Jaiswal-Nagar, S. Ramakrishnan, and A.K. Grover, Phys. Rev. B78, 134528 (2008).

30. K. Yamauchi, H. Katayama-Yoshida, A. Yanase, and H. Harima, Physica C412-414, 225 (2004).

31. M. Heinecke and K. Winzer, Z. Phys. B98, 147 (1995).

32. G. Goll, M. Heinecke, A.G. M. Jansen, W. Joss, L. Nguyen, E. Steep, K. Winzer, and P. Wyder, Phys. Rev. B53, R8871 (1996).

33. G. Goll, L. Nguyen, E. Steep, A.G.M. Jansen, P. Wyder, and K. Winzer, Physica B230-232, 868 (1997).

34. T. Terashima, H. Takeya, S. Uji, K. Kadowaki, and H. Aoki, Solid State Commun. 96, 459 (1995).

35. T. Terashima, C. Haworth, H. Takeya, S. Uji, H. Aoki, and K. Kadowaki, Phys. Rev. B56, 5120 (1997).

36. D. Bintley and P.J. Meeson, Physica C388-389, 181 (2003).

37. K. Koepernik and H. Eschrig, Phys. Rev. B59, 1743 (1999).

38. D.L. Bashlakov, Yu.G. Naidyuk, I.K. Yanson, G. Behr, S.-L. Drechsler, G. Fuchs, L. Schultz, and D. Souptel, J. Low Temp. Phys. 147, 335 (2007).

39. Yu.G. Naidyuk, O.E. Kvitnitskaya, I.K. Yanson, G. Fuchs, K. Nenkov, A. Walte, G. Behr, D. Souptel, and S.-L. Drechsler, Phys. Rev. B76, 014520 (2007).

40. S.-L. Drechsler, H. Rosner, I. Opahle, S.V. Shulga, and H. Eschrig, Physica C408-410, 104 (2004).

41. M. Michor, T. Holubar, C. Dusek, and G. Hilscher, Phys. Rev. B52, 16165 (1995).

42. T. Maniv, V. Zhuravlev, I. Vagner, and P. Wyder, Rev. Mod. Phys. 73, 867 (2001).

43. T.J.B.M. Janssen, C. Haworth, S.M. Hayden, P. Meeson, M. Springford, and A. Wasserman, Phys. Rev. B57, 11689 (1998).

44. K. Maki, Phys. Rev. B44, 2861 (1991).

45. T. Maniv, V. Zhuravlev, J. Wosnitza, O. Ignatchik, B. Bergk, and P.C. Canfield, Phys. Rev. B73, 134521 (2006). 